\title{
Authors' Reply to Verdelli et al.: There Is No Knowledge Without Experience
}

\author{
Francesca Ingegnoli • Athanasia Tourlaki • \\ Roberta Gualtierotti
}

Published online: 9 May 2014

(c) Springer International Publishing Switzerland 2014

We thank Dr. Verdelli and colleagues for their comments regarding our case report of the potential safe role of tocilizumab monotherapy in the treatment of a patient with rheumatoid arthritis (RA) and iatrogenic Kaposi sarcoma (KS) [1].

In the case reported by Dr. Verdelli and colleagues [2], KS arose under a treatment regimen of tocilizumab monotherapy. The two patients' characteristics are quite different. Our patient already had KS before tocilizumab, and he was naïve from potent immunosuppressants other than methotrexate [1]. By contrast, the other patient [2] developed 'ex novo' KS after receiving several immunosuppressants, traditional and biologic disease-modifying antirheumatic drugs before receiving tocilizumab, thus probably resulting in a more severe suppression of immune system response. Moreover, the patient described in this second report [2] is older (78 years old), and also developed psoriasis, suggesting a complex immune dysregulation.

It must be stressed that no relapse of KS was noted in our patient with RA treated with tocilizumab [1], although we cannot exclude that the natural history of KS in our patient would imply a remission of the tumor, independently from antirheumatic therapies. In our report [1], a key feature is the sustained remission of RA. The disease was severe in our case, and we had no further therapeutic options available apart from tocilizumab. Although no

F. Ingegnoli $(\bowtie) \cdot R$. Gualtierotti Division of Rheumatology, Department of Clinical Sciences and Community Health, University of Milano,

Piazza Cardinal Ferrari 1, Milan, Italy

e-mail: francesca.ingegnoli@unimi.it

\section{A. Tourlaki}

Dermatology Unit, Fondazione IRCCS Ca' Granda-Ospedale Maggiore Policlinico, Milan, Italy evidence exists for the safety of tocilizumab therapy in KS, there is a strong theoretical rationale for this treatment [3]. In fact, no relapse of KS was observed, and this is a very positive finding and an interesting solution to the conundrum the patient presented with.

In our opinion, this is a very intriguing topic, and as our understanding of the potential therapeutic targets increases, so too do the treatment options. There is considerable interest in expanding knowledge and clinical applications of tocilizumab; however, there are no published studies on KS. Although ours are the only two anecdotal cases reported, they may pave the way to further prospective studies.

Conflict of interest No sources of funding were used in the preparation of this manuscript. The authors state that they have no conflicts of interest.

\section{References}

1. Ingegnoli F, Tourlaki A, Gualtierotti R. Tocilizumab monotherapy in a patient with rheumatoid arthritis and iatrogenic Kaposi sarcoma. Clin Drug Investig. 2014;34:159-61.

2. Verdelli A, Bonciani D, Bonciolini V, Caproni M. Is tocilizumab safe in Kaposi sarcoma? A complex association among rheumatoid arthritis, psoriasis, and Kaposi sarcoma. Clin Drug Investig. 2014; doi:10.1007/s40261-014-0200-7.

3. An J, Lichtenstein AK, Brent G, et al. The Kaposi sarcomaassociated herpesvirus (KSHV) induces cellular interleukin 6 expression: role of the KSHV latency-associated nuclear antigen and the AP1 response element. Blood. 2002;99(2):649-54. 